\title{
A Multi-channel Multiple Access Scheme Using Frequency Offsets - Modelling and Analysis
}

\author{
Sarwar Morshed, Mitra Baratchi, Pranab K. Mandal, Geert Heijenk \\ University of Twente, The Netherlands \\ \{s.morshed, m.baratchi, p.k.mandal, geert.heijenk\}@utwente.nl
}

\begin{abstract}
Keywords-Multiple access technique, frequency-offset, multichannel S-Aloha, MAC protocol, TR-MAC.

Abstract-A system using frequency offset based transmitreference (TR) modulation allows multiple nodes to transmit simultaneously and asynchronously without any mutual timing coordination. Thus, such a system provides inherent capabilities for a multiple access in the medium access control (MAC) layer to coordinate the shared use of the common wireless medium among the nodes of the wireless sensor network (WSN). However, certain characteristics of a frequency offset based system limits its performance, for example, the number of available frequency offsets is limited as it depends on several system parameters, and the number of simultaneous communications using different frequency offsets is limited due to inter-user interference. In this paper, we introduce an extended version of the performance model of a basic slottedAloha system, that captures the basic phenomena of a multichannel system with a limited set of channels and a limit to the number of simultaneously used channels. An analysis of this model reveals the potential of a MAC protocol for TR modulation with frequency offsets.
\end{abstract}

\section{INTRODUCTION}

In wireless communications, the MAC protocol performs the multiple access control for the shared medium. Wireless sensor networks mostly use single channel systems for operation. However, throughput and capacity performance of single channel systems decline as the number of communicating nodes increases with the prevalence of internet of things (IoT). Thus, the WSNs need to have efficient multiple access scheme with multiple channels.

A multi-channel system attempts to increase the system throughput and efficiency by using multiple frequency channels. However, this performance improvement comes at the cost of increased power consumption, channel switching delay, channel coordination between sender and receiver, and adjacent channel interference [1]. The power consumption of a node increases if multiple transceivers are used to realize multi-channel communication. A single tunable transceiver to operate on different frequencies adds the overhead of channel switching delay. Most importantly, a detailed coordination for channel switching is necessary among the nodes to ensure that the receiver is on the same channel with the sender at the same time to receive the packet. Furthermore, multi-channel protocols also suffer from interchannel interference. However, there exists single channel systems that could offer performance like multi-channel system with limited complexity. TR modulation is one of such single channel systems.

Transmit-reference modulation offers frequency offsets, which can be used as virtual frequency channels for multiple access [2]. The sender using TR modulation modulates a reference signal with data, and sends both the modulated signal and the reference signal in the shared medium with a frequency offset known to the receiver. The receiver restores the transmitted data by correlating the received signal with a version of itself having the same frequency offset applied. In a TR system, the receiver only needs to know the frequency offset used by the sender in order to decode the received signal [3]. Thus, TR modulation offers a simplified solution to the channel coordination problem if nodes work in pairs. Moreover, multiple nodes can transmit simultaneously by employing several frequency offsets without the need for mutual timing coordination.

Exploiting this TR modulation in the physical layer, earlier the authors in [3-6] proposed a MAC layer protocol, called TR-MAC for low duty cycle and low data rate operation. The nodes using TR-MAC operate in pairs in synchronized link states by remembering the frequency offset to be used for future communication. As a preamble sampling protocol, the nodes using TR-MAC protocol perform independent duty cycling, and sleep most of the time to save energy. Furthermore, this duty cycling ensures that only few nodes communicate at a time, thus only few frequency offsets are used simultaneously. As a result, a single channel can be used for multiple access communication by different pairs of nodes using different frequency offsets. The communication is successful if different pairs of nodes within the range choose mutually exclusive frequency offsets. Multiple pairs of nodes choosing the same frequency offset within the range of a receiver would result in a collision, since the intended receiver would not be able to decode the transmitted signal properly. Thus, TR-MAC protocol exploits the frequency offset based TR modulation in the physical layer to provide more flexibility towards the upper layers.

The frequency offset based multiple access has two characteristics that limit its performance: i) The number of available frequency offsets gets limited, and depends on 
the desired data rate, symbol rate of the used modulation, and delay spread of the environment; and ii) The number of simultaneous communications using different frequency offsets becomes limited, since the receiver experiences problems to decode the signal within the increased interference level in the presence of several signals using different frequency offsets. Therefore, a suitable model for frequency offset based multiple access technique is needed that takes care of these limitations for coordinating the shared medium access in WSNs.

The main question addressed in this paper is: how do the multi-channel properties and the mentioned limitations affect the performance of multiple access control in a system based on transmit-reference modulation with frequency offsets? In this paper, we propose and analyze a model for multiple access scheme using frequency offsets. As our starting point, we take a basic model of single channel slotted Aloha (SAloha) [7]. We extend the single channel S-Aloha model for multi-channel S-Aloha with similar principles. In the multi-channel S-Aloha model, a node can randomly and independently choose any of the channels for a time slot to transmit a packet. A communication is successful if the chosen channel was unique for that time slot. Finally, we extend this model further, to take into account the specific limitations mentioned above. The result is a model that describes the behaviour of a slotted, Aloha-based access mechanism for WSNs based on transmit-reference modulation with multiple frequency offsets. As such, it provides fundamental insights into the performance limits of a MAC protocol for TR modulation.

The contributions of this paper are: i) we present a general model for a multi-channel slotted Aloha-based multiple access scheme where transmissions are successful if a unique channel is chosen, and if the total number of transmissions is below a certain limit; ii) we use the model to provide fundamental insight into the performance of a MAC protocol for transmit-reference modulation with frequency offsets; iii) as an intermediate product, we present a model for straight multi-channel slotted Aloha; and iv) we validate our models by comparing to results from a simulation model.

The remainder of this paper is organized in the following manner: Section II presents related work. Afterwards, the multiple access scheme using frequency offset model is given in Section III. Section IV presents the results and analysis in terms of efficiency and throughput of the system. Finally, Section V provides the concluding remarks of our work, and suggests the future work.

\section{RELATED WORK}

In wireless communication, medium access control has been analyzed for several decades. Various multiple access protocols are available in the literature [8], [9]. Aloha was the first multiple access protocol that allows a node to transmit a packet as soon as it receives the packet from the upper layers [10]. In case of a collision, the senders retransmit the packet with a certain probability $p$. With this simple mechanism, Aloha provides a maximum of $18 \%$ channel efficiency. Afterwards, S-Aloha was proposed where a channel is divided in several time slots, and nodes defer the packet transmission to the start of a time slot [7]. The maximum efficiency of S-Aloha is $37 \%$ after using the time slot based mechanism.

A generic multi-channel system consists of several independent channels. To transmit a packet, a user can choose any of these channels using a suitable multiple access protocol. A simple multi-channel system could be a multichannel slotted-Aloha system that consists of multiple equalcapacity slotted channels shared by several users, where a user first chooses a channel, then chooses a slot to transmit a packet. A multi-channel S-Aloha protocol has been studied in [11], and a stability optimization criterion with and without receiver collision has been studied in [12]. This multi-channel system improved performance, and offered cost reduction and fault tolerance compared to the single channel system. The performance of multi-channel slottedAloha system was also analyzed in [13] and [14] with a deterministic channel model without multi-path fading. The authors in [15] discussed about stabilizing the multi-channel S-Aloha from the context of M2M communication.

The main difference of this research with the other research is that we propose a model of an intermediate multichannel S-Aloha, and use the channel selection mechanism of that intermediate multi-channel S-Aloha to propose a novel multiple access model using frequency offsets.

\section{ModelLING MULTIPLE ACCESS SCHEMES}

In this section, we present models of existing multiple access schemes, propose a new frequency offset based multiple access technique, and extend the presented models to capture its behaviour. The multiple access problem deals with the coordination of multiple sending and receiving nodes sharing a common medium. We start with the basic multiple access scheme of S-Aloha for a single channel. Afterwards, we model a simple multiple access scheme for multiple channels using S-Aloha. Finally, we model the multiple access technique with multiple frequency offsets.

\section{A. S-Aloha system}

S-Aloha is a simple multiple access technique for a single channel, where the channel is divided into time slots. A node always transmits in the beginning of a time slot. Whenever a node receives a packet to transmit, the transmission is delayed until the start of the next time slot [10]. A transmission can be successful if only one packet is present in a slot. Collision happens in the presence of multiple packets in a time slot. Thus, the vulnerable period of S-Aloha is essentially the time slot duration, where the 
desired packet can collide with transmissions coming from other transmitting nodes.

In modelling S-Aloha, it is usually assumed that the probability $p$ that a certain node transmits within a time slot for a fresh packet is the same for a retransmitted packet that suffered a collision. Hence, the average number of packet transmissions in the system within a time slot can be given by $\lambda=n p$, where $n$ is the number of active nodes. Let us now define the random variable $K$ as the number of nodes that are transmitting simultaneously. If we assume the packet arrival process is a Poisson process, the probability of $k$ arrivals, or equivalently $k$ transmission attempts, within a unit time is given by

$$
P(K=k)=e^{-\lambda} \frac{\lambda^{k}}{k !} .
$$

Therefore, the average number of packets that can be successfully delivered, i.e., the throughput, $S_{S}$, for single channel S-Aloha system is given by

$$
\begin{aligned}
S_{S} & =\mathbb{E}(\text { No.of successful transmissions per slot }) \\
& =P(K=1)=\lambda e^{-\lambda} .
\end{aligned}
$$

S-Aloha only provides successful transmissions without collision when only one transmission attempt is made per time slot. The efficiency of the S-Aloha system would be the same as its throughput because of the availability of only one channel, and can be given by

$$
E_{S}=\lambda e^{-\lambda} \text {. }
$$

\section{B. Multi-channel S-Aloha system}

In this section, we extend the model of S-Aloha for multiple channels. The working principle of multi-channel S-Aloha would be similar to that of S-Aloha with $C$ narrowband S-Aloha channels in parallel occupying the same bandwidth as the S-Aloha system. In this way, the collision domain would be limited to that particular channel. A node would select a channel randomly and independently of other nodes to initiate a packet transmission. The communication would be successful if one node chooses a unique channel for a single time slot. Collision happens if multiple transmitters choose the same channel for a single time slot.

Therefore, the system throughput, $S_{M}$, for a multi-channel S-Aloha system with $C$ channels can be derived as

$$
\begin{aligned}
S_{M}= & \mathbb{E}(\text { No. of successful transmissions per timeslot }) \\
= & \sum_{k=1}^{\infty} P(K=k) \\
& \times \mathbb{E}(\text { No. of succ. trans. per timeslot } \mid K=k) \\
= & \sum_{k=1}^{\infty} P(K=k) \sum_{s=1}^{k} s P(\text { s out of } k \text { succ. trans. }) \\
= & \sum_{k=1}^{\infty}\left(e^{-\lambda} \frac{\lambda^{k}}{k !}\right) \sum_{s=1}^{\min (k, C)} s P(C, k, s),
\end{aligned}
$$

where $P(C, k, s)$ denotes the probability of $s$ successful transmission when $k$ nodes are transmitting simultaneously, and $C$ channels are available. In Eq. 4, we have also used the fact that the number of successful transmissions $(s)$ cannot be more than the number of attempts $(k)$ or the number of channels $(C)$ present. The formula to calculate $P(C, k, s)$ is given later in this section.

The efficiency of the multi-channel S-Aloha system can be derived by normalizing the throughput by the number of available channels, $C$, and can be given by

$$
\mathbb{E}_{M}=\frac{1}{C} \sum_{k=1}^{\infty}\left(e^{-\lambda} \frac{\lambda^{k}}{k !}\right) \sum_{s=1}^{\min (k, C)} s P(C, k, s) .
$$

\section{Multiple access scheme using frequency offsets}

In a system with transmit-reference modulation, the multiple access scheme can be based on multiple frequency offsets, where different nodes use different frequency offsets to communicate with each other. To transmit a packet, one node can randomly choose a frequency offset from the frequency offset pool, and modulate the carrier signal with the chosen frequency offset. The receiver can use the same frequency offset to decode the received signal in order to retrieve the original transmitted data. Therefore, the frequency offset pool can be referred to as a collection of virtual channels.

In a frequency offset based multiple access system, a node could independently and randomly choose a frequency offset to initiate a packet transmission. A transmission is successful only if the frequency offset chosen by one node is not chosen by any other node within its range at the same time. Collision occurs if another node within the coverage area chooses the same frequency offset to transmit simultaneously. As a result, a multiple access scheme using frequency offsets can be modelled using the similar principles of multi-channel S-Aloha. Furthermore, now $C$ corresponds to the number of maximum available frequency offsets for multiple access with frequency offset. The number of unique frequency offsets in the offset pool is variable, and depends on the system parameters, for example, data rate, coherence bandwidth, and delay spread of the environment. As a result, the total number of unique frequency offsets available for use gets limited. A calculation of the number of frequency offsets based on the system parameters is given in Appendix A.

However, there is an important difference. Unlike in multichannel S-Aloha system, the number of simultaneous communications is limited with a maximum value of $m$, due to the limit in acceptable interference level. Assuming a scenario where multiple transmissions using several different frequency offsets are destined to different receivers. The total interference level is increased by these simultaneous transmissions because of the cross-mixing among signals from different active nodes using different frequency offsets. The authors in [2] analyzed the limitation on simultaneous 
communications for a desired receiver in the presence of several simultaneous transmissions, and concluded at most 3 to 4 active transmissions within the acceptable interference level. It may even be possible to have fewer parallel transmissions towards the same receiver, which is out of scope of this paper.

We can then derive the system throughput, $S_{F O}$, for a frequency offset based multiple access scheme with $C$ available frequency offsets, and maximum $m$ number of simultaneous communications as

$$
\begin{aligned}
S_{F O} & =\sum_{k=1}^{\infty} P(K=k) \mathbb{E}(\text { No. of succ.trans. } \mid K=k) \\
& =\sum_{k=1}^{m} P(K=k) \mathbb{E}(\text { No. of succ.trans. } \mid K=k),
\end{aligned}
$$

since with more than $m$ simultaneous communication attempts, none can be successful. This can be further simplified, similar to Eq. (4), as

$$
S_{F O}=\sum_{k=1}^{m}\left(e^{-\lambda} \frac{\lambda^{k}}{k !}\right) \sum_{s=1}^{\min (k, C)} s P(C, k, s) .
$$

The efficiency of the frequency offset based multiple access system can be derived by normalizing the throughput by the number of maximum allowed simultaneous communication limitation, $m$, and can be given by

$$
\mathbb{E}_{F O}=\frac{1}{m} \sum_{k=1}^{m}\left(e^{-\lambda} \cdot \frac{\lambda^{k}}{k !}\right) \sum_{s=1}^{\min (k, C)} s P(C, k, s) .
$$

The success probability, $P(C, k, s)$, is derived based on the condition where $s$ number of simultaneous communications become successful out of $k$ simultaneous transmission attempts by choosing unique frequency offsets out of $C$ frequency offsets. Recall that a node will be successful in transmitting only if the frequency offset chosen by the node is unique in the sense that it is not chosen by any other node. Since the total number of ways $k$ nodes can choose from $C$ frequency offsets is $C^{k}$, and each node chooses a frequency offset randomly and independently of each other, the success probability, $P(C, k, s)$, is given by

$$
P(C, k, s)=\frac{1}{C^{k}} f(C, k, s) .
$$

Here $f(C, k, s)$ provides the number of possible ways $k$ nodes can choose from $C$ frequency offsets with exactly $s$ nodes having selected a unique frequency offset. Note that exactly $s$ nodes select a unique frequency offset means that none of the remaining $(k-s)$ nodes select a unique frequency offset from the remaining $(C-s)$ frequency offsets. If $\left(\begin{array}{l}k \\ s\end{array}\right)$ represents the possible sets of nodes that can be successful, and the number of $s$ permutations of $C,{ }^{C} P_{s}$, represents the possible ways this set of $s$ nodes can choose unique frequency offsets from $C$ frequency offsets, then we can write $f(C, k, s)$ as

$$
f(C, k, s)=\left(\begin{array}{l}
k \\
s
\end{array}\right){ }^{C} P_{s} f(C-s, k-s, 0) .
$$

Now we derive an expression for $f\left(C^{*}, k, 0\right)$ that represents the number of ways $k$ nodes can select frequency offsets from $C^{*}$ frequency offsets, and none having a unique frequency offset. This happens when any of the frequency offsets chosen by a node is also chosen by at least another node. This resembles to situations where the nodes form groups of size two or more, then choose a frequency offset as a group. For example, one possibility is when all of the nodes choose the same frequency offset, which is equivalent to all the nodes forming one group, then as a group choosing a frequency offset. Another possibility is that few of the nodes choose the same frequency offset as one group, and the rest of the nodes choose another frequency offset as another group. Since each group must contain at least two nodes, the maximum number of groups that can be formed is $\lfloor k / 2\rfloor$. We use 2-associated Stirling number of second kind [16], $S_{2}(k, i)$, that represents the number of possible group formations from $k$ distinguishable objects into $i$ groups each containing at least 2 objects. The number of ways these $i$ groups can choose from $C^{*}$ frequency offsets is represented by ${ }^{*} P_{i}$. Hence, $f\left(C^{*}, k, 0\right)$ can be represented as

$$
f\left(C^{*}, k, 0\right)=\sum_{i=1}^{\lfloor k / 2\rfloor} S_{2}(k, i){ }^{C^{*}} P_{i} .
$$

\section{RESUlts AND ANALYSis}

We implement the analytical models of the frequency offset based multiple access and the multi-channel S-Aloha in Matlab. We also simulate the frequency offset based system using discrete event simulator OMNeT++. For the simulation, we consider a single server with many interfaces that can represent the multiple virtual channels using frequency offsets. One node randomly chooses a frequency offset, a virtual channel in this case, to communicate with the server. In the server, we impose the limitation on simultaneous communications imposed by the TR modulation using frequency offsets. The server concludes successful packet reception if different packets are received in different virtual channels for a single time slot within the permitted limitation on the number of simultaneous communications. In the rest of the section, we present the results for both of these models in terms of the total throughput and the efficiency while varying the offered load. For frequency offset based multiple access model, we consider different limitations on simultaneous communications. For statistical accuracy, 100 simulation runs are averaged with $95 \%$ confidence intervals. Afterwards, we present the analytical results for multi-channel S-Aloha model, that we developed as an intermediate step. 


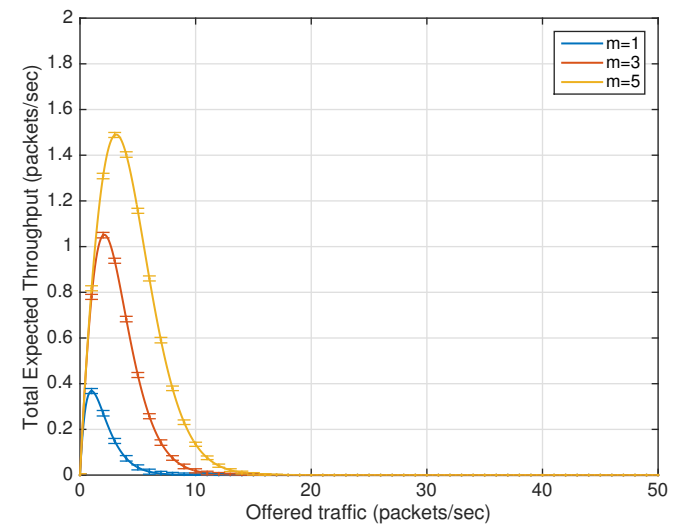

a) No. of frequency offsets, $\mathrm{C}=5$

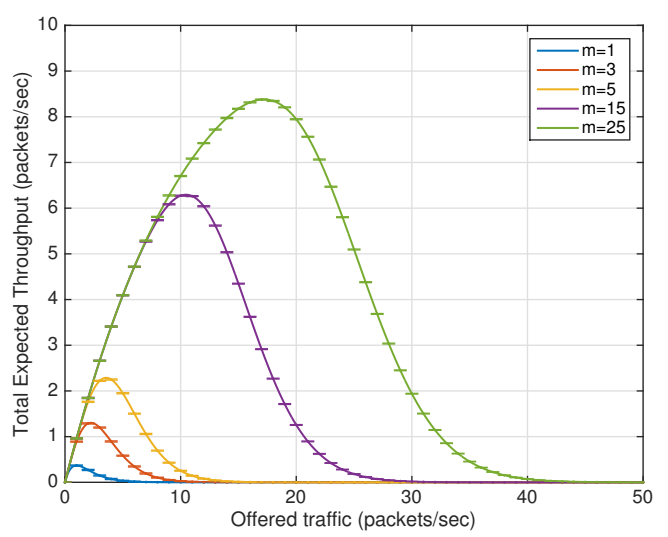

b) No. of frequency offsets, $\mathrm{C}=25$

Figure 1: Throughput of the frequency offset based system

\section{A. Performance of frequency offset based multiple access}

In this subsection, we present the performance evaluation results in terms of the throughput and the efficiency for the frequency offset based multiple access scheme for different limitation on the maximum number of simultaneous transmissions, $m$, within the acceptable interference level. The throughput and the efficiency results are obtained while varying the offered traffic for analytical modelling using Matlab, as presented in Section III, and simulation results using OMNeT++. Here we present the results for frequency offsets $C=5$ and $C=25$ to show how the throughput and the efficiency changes, when the number of frequency offsets in the system is increased from $C=5$ to $C=25$. We choose $C=25$, since this is a realistic value for the number of frequency offsets with the given parameters, as calculated in the Appendix A.

$i$. Throughput: The throughput results for frequency offset based multiple access model is given in Fig. 1 with respect to the offered traffic variation for frequency offsets $C=5$ and $C=25$, as presented in Section III Eq. 6. Here we see a significant linear increase of throughput at offered traffic load close to zero with increasing the number of simultaneous transmissions, $m$. After reaching the maximum, the throughput decreases with increasing traffic load, due to many collisions caused by the overload of the system. Furthermore, by comparing the throughput of frequency offsets $C=5$ and $C=25$, we see a significant increase in the total throughput of the system, when more offsets are added to the system. Note that the scaling in y-axis for throughput results are different for frequency offsets of $C=5$ and $C=25$ cases. The simulation results with $95 \%$ confidence interval also confirm the analytical results.

ii. Efficiency: We present the efficiency results for the frequency offset based multiple access scheme for different limitations on the maximum number of simultaneous transmissions, $m$, in Fig. 2. To calculate efficiency, we normalize the throughput with respect to the number of simultaneous transmissions allowed, as presented in Section III Eq. 7. At offered load close to zero, we see linear increase of efficiency that slows down after reaching a maximum point with increasing traffic load, as seen for the throughput case.

Here we see that the shapes of the curves correspond to the characteristic behaviour of slotted Aloha. We observe that $m=1$ results in the single channel S-Aloha system, because only one simultaneous communication is allowed. This also provides basic validation of our model as a special case. As $m$ increases for a small number of frequency offsets, for example $C=5$, the maximum efficiency decreases; since increasing the limitation on simultaneous communications reduces the efficiency. However, we observe that the maximum efficiency increases when the number of frequency offsets increases, for example $C=25$, and with a relatively low number for the limitation on simultaneous communications, for example $m=5$. The reason is that the probability of having a successful communication increases, when more offsets become available to choose from the frequency offset pool. As a result, we observe the maximum efficiency exceeds the theoretical maximum of S-Aloha for a higher number of frequency offsets available in the pool, when a moderately low number of simultaneous communications are allowed. The maximum efficiency eventually decreases with imposing higher limitations on the number of simultaneous communications, for example $m=25$, with increasing traffic load.

\section{B. Performance of multi-channel S-Aloha}

As an intermediate step, we have obtained a model for multi-channel S-Aloha. Here we present its performance in terms of efficiency for several number of available channels while varying the offered load in Fig. 3, as presented in the analytical model in Section III Eq. 5. We see that the 


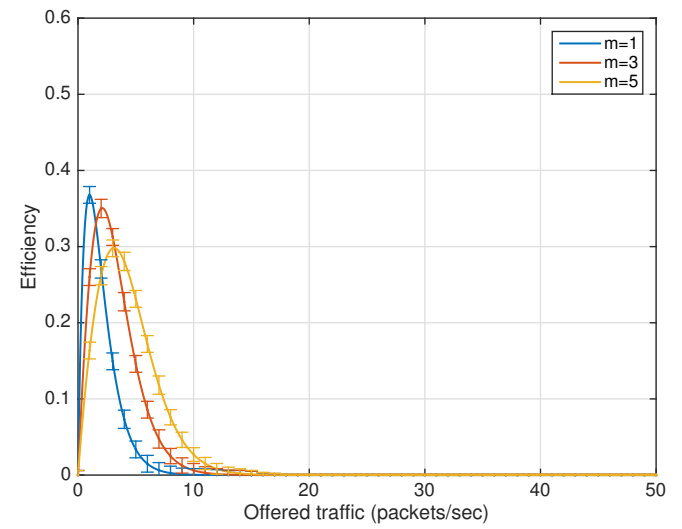

a) No. of frequency offsets, $\mathrm{C}=5$

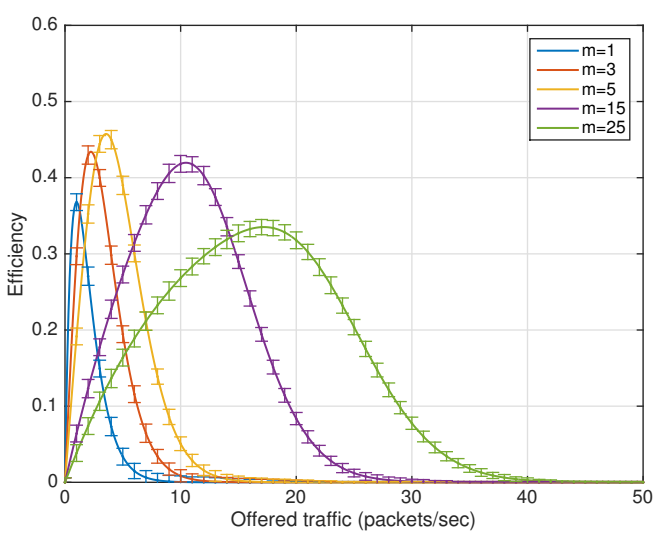

b) No. of frequency offsets, $\mathrm{C}=25$

Figure 2: Efficiency of the frequency offset based system

shapes of the curves correspond to the multi-channel SAloha. When a single channel is available for $C=1$, the system successfully corresponds to the single channel S-Aloha system along with its maximum efficiency. As the number of frequency offsets increases, the efficiency maintains the maximum efficiency value. Furthermore, the position of the maximum efficiency point corresponds to the offered load, that is, $C=25$ provides highest efficiency when offered load is 25 packets/sec. It means that a multichannel system with 25 channels provides its highest efficiency, when 25 packet transmissions are taking place in 25 different channels. Therefore, the results correspond to what can be intuitively expected.

\section{CONClusions AND Future Work}

This paper proposes a model for multiple access for frequency offset based transmit-reference modulation using a general model of multi-channel S-Aloha based multiple

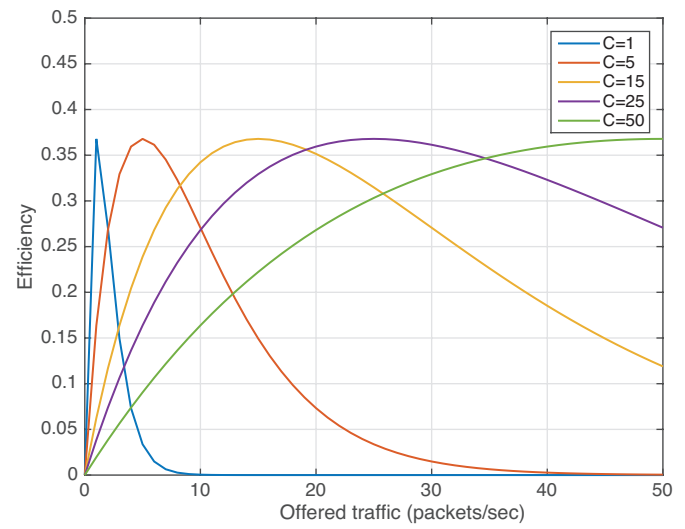

Figure 3: Efficiency of multi-channel S-Aloha

access, where a successful communication implies that a unique offset is chosen, and the total number of simultaneous communications is below a certain limit. This multiple access model provides the fundamental insight into the performance of a MAC protocol that exploits TR modulation in the physical layer. We find that the frequency offset based system provides much higher throughput and an increased efficiency compared to the multi-channel S-Aloha system under similar conditions. For our future work, we will analyze the multiple access protocol utilizing carrier sense with total interference level, and taking duty cycle operation into account. The total interference level increases by simultaneous transmissions and the cross-mixing among signals from different active nodes using different frequency offsets.

\section{APPENDIX}

\section{A. Frequency Offset calculation}

Using frequency offset based transmit-reference modulation system, only a discrete set of frequency offsets are usable. The reason is, a frequency offset must be an integer multiple of symbol rate, $R_{b}$ [2], which is equivalent to the data rate for binary phase shift keying (BPSK) modulation. However, the physical layer imposes a restriction on the maximum number of usable frequencies to be used as a frequency offset, represented by $f_{\max }$. This maximum frequency has to be much smaller than the coherence bandwidth of the channel, $B_{C}$; i.e., $f_{\max } \ll B_{C}$ to ensure the reference and the information signals are affected similarly by the channel [2]. As a rule of thumb, this condition translates as $f_{\max } \leqslant B_{C} / 10$. The coherence bandwidth is inversely proportional to the delay spread, $\tau_{r m s}$, which has typical value of $100 \mathrm{nsec}$ for an indoor office environment [17]. Therefore, the maximum frequency to be used as an offset, 
$f_{\text {max }}$, in an office environment is given by

$$
f_{\text {max }} \leqslant \frac{B_{C}}{10} \leqslant \frac{\frac{1}{\tau_{r m s}}}{10} \leqslant \frac{\frac{1}{100 n s}}{10} \leqslant 1000 K H z .
$$

All the frequency offsets to be used should be less than the maximum frequency. For a data rate of $25 \mathrm{kbps}$, the corresponding symbol rate becomes $25 \mathrm{KHz}$. Thus, the number of available frequency offsets, $N_{F}^{A}$, is given by

$$
N_{F}^{A}=\frac{f_{\max }}{R_{b}}=\frac{1000 \mathrm{KHz}}{25 \mathrm{KHz}}=40 .
$$

However, the frequency offset in a particular link should not be twice the frequency offset of another simultaneous link [2], that is, $f_{i} \neq 2 f_{j}$ for $i \neq j$. This condition is vitally important to minimize the inter-user interference and maximize the signal-to-noise ratio. As a result, the number of usable frequency offsets, $N_{F}^{U}$, is given by

$$
\begin{gathered}
N_{F}^{U}=\frac{N_{F}^{A}}{2}+\sum_{d=1}^{d_{\max }} \Gamma\left(\frac{N_{F}^{A}+4^{d}}{2^{2 d+1}}\right), \text { for even } N_{F}^{A}, \\
N_{F}^{U}=\frac{N_{F}^{A}+1}{2}+\sum_{d=1}^{d_{\max }} \Gamma\left(\frac{N_{F}^{A}+4^{d}}{2^{2 d+1}}\right), \text { for odd } N_{F}^{A} .
\end{gathered}
$$

Here $\Gamma(a / b)$ is the quotient of the division of the variable $a$ by $b$, and $d_{\max }$ is the greatest dividing exponent of a base 4 with respect to $N_{F}^{A}$, i.e., $4^{d_{\max }} \leq N_{F}^{A}$, and $4^{d_{\max }+1}>N_{F}^{A}$. As $N_{F}^{A}$ is 40 , thus $d_{\max }$ is 2 for this case $\left(4^{2} \leqslant 40\right.$ and $\left.4^{3}>40\right)$. Since $N_{F}^{A}$ in our case is an even number 40 , we can deduce using Eq. 13 that for a data rate of $25 \mathrm{kbps}$, at most 26 frequency offsets are usable in simultaneous communications within the 40 available frequency offsets.

\section{ACKNOWLEDGEMENT}

This work is supported by the Dutch Technology Foundation (STW) through the WALNUT project (no. 11317). Special thanks to Ibrahim Bilal of Telecommunication Engineering group from University of Twente, The Netherlands for his contribution in the Appendix.

\section{REFERENCES}

[1] O. D. Incel, "Survey paper: A survey on multi-channel communication in wireless sensor networks," Comput. Netw., vol. 55, pp. 3081-3099, Sept. 2011.

[2] B. I. Bitachon, I. Bilal, A. Meijerink, and M. J. Bentum, "Near-far problem in noise-based frequencyoffset modulation," in Communications and Vehicular Technology in the Benelux (SCVT), 2015 IEEE Symposium on, pp. 1-6, Nov 2015.

[3] S. Morshed and G. J. Heijenk, "TR-MAC: an energyefficient MAC protocol for wireless sensor networks exploiting noise-based transmitted reference modulation," in 2nd Joint ERCIM eMobility and MobiSense
Workshop, St. Petersburg, Russia, pp. 58-71, June 2013.

[4] S. Morshed and G. Heijenk, "TR-MAC: An energyefficient MAC protocol exploiting transmitted reference modulation for wireless sensor networks," in Proceedings of the 17th ACM International Conference on Modeling, Analysis and Simulation of Wireless and Mobile Systems, MSWiM '14, pp. 21-29, ACM, 2014.

[5] S. Morshed and G. J. Heijenk, "Optimization and verification of the tr-mac protocol for wireless sensor networks.," in WWIC, vol. 9071 of Lecture Notes in Computer Science, pp. 396-410, Springer, 2015.

[6] S. Morshed, M. Baratchi, and G. Heijenk, "Trafficadaptive duty cycle adaptation in tr-mac protocol for wireless sensor networks," in 2016 Wireless Days (WD), pp. 1-6, March 2016.

[7] L. G. Roberts, "Aloha packet system with and without slots and capture," SIGCOMM Comput. Commun. Rev., vol. 5, pp. 28-42, Apr. 1975.

[8] R. Rom and M. Sidi, Multiple access protocols : performance and analysis. Telecommunication networks and computer systems, New York: Springer-Verlag, 1990.

[9] D. Bertsekas and R. Gallager, Data Networks (2Nd Ed.). Upper Saddle River, NJ, USA: Prentice-Hall, Inc., 1992.

[10] N. Abramson, "THE ALOHA SYSTEM: another alternative for computer communications," in AFIPS '70 (Fall): Proceedings of the November 17-19, 1970, fall joint computer conference, (New York, NY, USA), pp. 281-285, ACM, 1970.

[11] W. Szpankowski, "Packet switching in multiple radio channels: Analysis and stability of a random access system.," Computer Networks, vol. 7, pp. 17-26, 1983.

[12] I. E. Pountourakis and E. D. Sykas, "Analysis, stability and optimization of aloha-type protocols for multichannel networks," Comput. Commun., vol. 15, pp. 619629, Dec. 1992.

[13] W. Yue, "The effect of capture on performance of multichannel slotted ALOHA systems," IEEE Trans. Communications, vol. 39, no. 6, pp. 818-822, 1991.

[14] Z. Zhang and Y. Liu, "Comments on 'the effect of capture on performance of multichannel slotted ALOHA systems',' IEEE Trans. Communications, vol. 41, no. 10, pp. 1433-1435, 1993.

[15] O. Galinina, A. Turlikov, S. Andreev, and Y. Koucheryavy, "Stabilizing multi-channel slotted aloha for machine-type communications," in Information Theory Proceedings (ISIT), 2013 IEEE International Symposium on, pp. 2119-2123, July 2013.

[16] "Oeis foundation inc. (2011), the on-line encyclopedia of integer sequences,", http://oeis.org/A008277.

[17] A. Molisch, Wireless Communications. Wiley-IEEE Press, 2005. 